Research Article

\title{
Effects of Southwest Airlines on Carrier Profits and Entry Probabilities
}

\author{
Junqiushi Ren \\ Research Institute of Economics and Management, Southwestern University of Finance and Economics, Chengdu 611130, China \\ Correspondence should be addressed to Junqiushi Ren; junqiushiren@yahoo.com
}

Received 23 December 2020; Revised 12 March 2021; Accepted 8 April 2021; Published 19 April 2021

Academic Editor: Aditya Rio Prabowo

Copyright (c) 2021 Junqiushi Ren. This is an open access article distributed under the Creative Commons Attribution License, which permits unrestricted use, distribution, and reproduction in any medium, provided the original work is properly cited.

\begin{abstract}
This paper studies the effects of Southwest Airlines, the largest low-cost carrier (LCC) in the U.S., on other carriers' payoff functions and entry probabilities. A static entry game model is developed and estimated by viewing entry as an indicator of underlying profitability and making use of Nash Equilibrium. Results indicate that Southwest has a remarkable and negative impact on the payoffs of other carriers. This impact is firm-specific, with LCCs being more affected than full-service carriers (FSCs). Comparing the two service types, the results show that Southwest's nonstop presence apparently imposes more downward pressure on opponents' profits than its connecting presence. A counterfactual experiment is then conducted. Once Southwest is counterfactually removed, the probability of each carrier entering a market significantly changes. This paper examines Southwest's impacts from a new perspective and extends literature on entry game estimation.
\end{abstract}

\section{Introduction}

The United States commercial airlines can be divided into full-service carriers (FSCs) and low-cost carriers (LCCs). Compared to FSCs, the key competitive advantage of LCCs is their ability to offer significantly lower fares through highly efficient cost-cutting strategies such as point-to-point transit for higher aircraft utilization, shorter turnaround time, higher seating density, and operating uniform fleets. Over the past few decades, such low-cost-low-fare strategy has enabled LCCs to grow rapidly. In 2017, $49 \%$ of U.S. domestic passengers travelled with LCCs, where Southwest Airlines, the leading LCC, itself transported $27.9 \%$ of domestic traffic, making it the largest LCC in the U.S. and across the world (see [1]).

Given the fact that LCCs have been so successful, it is not surprising that they have attracted a lot of attention in the literature. A great deal of research has been conducted to demonstrate LCCs' dramatic downward pressure on airfares, which, in turn, leads to a significant increase in air travel demand and passenger traffic (examples include [2-9] and many others). Nevertheless, with a concurrent fare drop and overall traffic rise, it is unknown whether and to what extent LCCs may affect other carriers' profits on a route. Consequently, it is also unclear how LCCs may influence their probabilities of entering a route.

This paper aims to study how Southwest affects other carriers' payoff functions and entry probabilities. Empirically, there are at least three difficulties in this estimation. First, each carrier's payoff function should be private information and only partly observable to researchers. Second, each carrier's entry choice should be an endogenous action which is determined by expected profit. This endogeneity must be reflected in the estimation. Third, a carrier's expected profit and entry choice should be affected by other carriers' entry choices, meaning that their decisions influence each other. Such strategic interaction is an important determinant and should be captured. In order to address those problems as much as possible, this paper constructs a game model similar to that in $[10]$ and then estimates the parameters using the bounds method proposed in [11].

In particular, this paper develops a static entry model for the U.S. airline market. Assuming entry decisions are an indicator of underlying profitability, the parameters in the payoff functions are recovered such that model predicted entry outcomes match actual entry outcomes. Based on the 
estimated payoff functions, a counterfactual experiment is then performed to assess Southwest's impacts on other carriers' entry probabilities.

The results show that Southwest has a substantial and negative impact on the payoff functions of other carriers. This impact is firm-specific, with LCCs being more affected than FSCs. Comparing the two service types, the results indicate that Southwest's nonstop presence apparently imposes more downward pressure on opponents' profits than its connecting presence. Finally, by conducting counterfactual experiment, the results show that removing Southwest increases the entry probability of each carrier.

One may doubt why a game-theoretic approach is adopted here rather than a machine-learning approach. Game theory was originally invented to explain interactive economic behaviors. In the context of this article, airlines make interactive decisions to maximize profits, which closely match with game theory economic rationale. As a result, a game-based approach could better reveal airlines' decision-making logics and, more importantly, provide economic interpretations.

The remaining portion of this paper is organized as follows. Section 2 reviews relevant literature. Section 3 introduces the data and the steps taken to construct the sample. Section 4 explains the game and the empirical model. Section 5 describes the main results. Concluding remarks are made in Section 6.

\section{Related Literature}

This paper is related to three streams of literature, namely, literature on LCCs, literature on game-theoretic analysis for networking, and literature on entry game estimations.

2.1. Literature on LCCs. It has long been believed that the introduction of LCCs can cause a drop in airfares. As early as 1993, Bennett and Craun concluded that Southwest's operations on the Oakland-Burbank route resulted in a 55\% decrease in prices. Later research studies found similar results by studying different routes and different settings, including $[3-9,12]$ and many others.

Meanwhile, it has been well recognized that the price drops are accompanied with significant increases in air travel demand and passenger traffic. For example, [13] showed that there was a six-fold increase in passenger volume following Southwest's entry; [14] documented a traffic increase of $200 \%$ on routes entered by Southwest; [5] controlled fixed effects and found that the magnitude of traffic increases is roughly twice that of the fare declines; and [2] showed that the entry or growth of LCCs at US airports clearly stimulated passenger volumes.

Nonetheless, with a concurrent fare drop and overall traffic rise, it is unclear whether and to what extent LCCs may affect other carriers' profits on a route. As a result, it is also unclear how LCCs may influence their probabilities of entering a route.

Interestingly, although the impacts of LCCs on airfares and passenger traffic volumes have been extensively studied, research into their impacts on carriers' profits or entry probabilities is sporadic. To the best of the author's knowledge, [15] is the only paper that examined the effect of LCC's entry on profits, which demonstrated a decrease in FSC's profit by calculating profit from the carrier's average yields and unit cost, route distances, and the number of passengers. Unlike [15], the present paper studies this question by recovering a profit index from games and investigates the changes of profit from a different perspective.

\subsection{Literature on Game-Theoretic Analysis for Networking.} This paper borrows its theoretical framework from classic literature on game-theoretic analysis for networking. Specifically, [10] provides a systematic introduction to the application of game theory in the field of wireless networking, which motivated the author for the analysis framework of this article.

2.3. Literature on Entry Game Estimations. The estimation approach of this article is closely related to the literature on empirical entry games. Originated in [16-18], scholars have made important steps of estimating entry games applying the insight that observed entry decisions should be an indicator of underlying profitability. However, it has also been recognized that the existence of multiple equilibria constitutes a big challenge in estimating this kind of models. As an example, suppose there is a market with three potential firms: one large and two small. The possible market structure could be one where the large firm has a monopoly, but it could also be a duopoly of the two smaller firms. To deal with multiplicity, Berry and Bresnahan and Reiss [16, 17] proposed to make equilibrium selection assumptions [11] and, on the other hand, developed a more general framework to estimate the upper and lower bounds of parameters without manually selecting an equilibrium.

This paper closely follows the bounds method provided in [11], but different from it in the following important aspects. First, while Ciliberto and Tamer [11] restricted their attention to nonstop flights, the present article extends the analysis to incorporate both nonstop and connecting flights. Including both types of services may be important here since Reiss and Spiller [19] concluded that the type of services is an essential determinant of the level of competition, and Dunn [20] further showed that service types are important factors influencing airlines' decisions. Second, while the purpose of [11] was to develop the bounds approach and prove its identification, the focus of this paper is to apply the technique to investigate Southwest's impacts. Third, the data used in [11] come from 2001Q2, whereas this paper uses data from 2017Q1, which is also important since the market has undergone tremendous changes over the years.

As a brief summary, this paper adds to the literature in three major ways. First, although massive research studies have been conducted to assess the effects of LCCs on airfares and passenger traffic, their impacts on carrier profits or entry probabilities are rarely explored. This paper fills the gap. Second, it extends literature on empirical entry games by being the first work to incorporate both nonstop and 
connecting services into the model. Last but not the least, the findings of this paper indicate that nonstop service and connecting service compete with each other in a very asymmetric way, which provides useful guidance to antitrust practices.

\section{Data}

3.1. Data Sources. The primary data employed in this study come from the Airline Origin and Destination Survey (DB1B) (available at https://www.transtats.bts.gov/Tables. asp?DB_ID=125\&DB_Name=Airline $\% 20$ Origin $\% 20$ and $\%$ 20Destination $\% 20$ Survey\%20\%28DB1B\%

29\&DB_Short_Name=Origin\%20and\%20Destination\% 20Survey), which are collected and published by the Office of Airline Information of the US Bureau of Transportation Statistics. The data are quarterly data that consist of a $10 \%$ random sample of all tickets sold and operated by US carriers.

The sample used in this study includes data in the first quarter of 2017. That being said, this study is based on a cross-sectional dataset in order to construct a static game. For each trip in this quarter, the following information is collected: the reporting carrier, the transaction price, the number of passengers, the airport sequence (origin and destination airports along with all transfer points), the actual flight distance, and the nonstop flight distance between the two endpoint airports.

The following data restrictions are then imposed: (i) drop trips that are part of international travel; (ii) drop trips that involve more than one intermediate transfer point to simplify the analysis; (iii) drop trips with fares less than $\$ 25$ due to the high probability that those may pertain to frequentflyer programs where passengers redeemed accumulated travel points to offset the full fare of travel (this is common in the literature; see, for example, [21, 22]); and (iv) drop codeshare trips.

In addition to $\mathrm{DB} 1 \mathrm{~B}$, the author also collects demographic statistics including population and income for all the metropolitan statistical areas (MSAs) of the United States from the U.S. Census Bureau.

3.2. Data Aggregation. The DB1B data are aggregated into market level in two steps. First off, the author summarizes data into "market-carrier-service type" cells, where a "market" is characterized by the two endpoint airports, independent of any intermediate transfer stops and the heading of the flight (the definition of market follows $[5,11,23])$; "carrier" refers to the reporting carrier; and "service type" is either nonstop or connecting. To illustrate, the cell "JFK-SAN-Delta-nonstop" consists of all nonstop trips between New York John F. Kennedy International Airport and San Diego International Airport reported by Delta Air Lines.

The author then computes the total number of passengers within each cell and drops the cells whose total number of passengers is fewer than 90 (for a predicted quarterly traffic of 900 , which corresponds to a once-a-week flight by a medium-size jet) in order to get rid of very unpopular combinations.

Subsequently, the author aggregates the data by market segments. In doing so, the author creates several dummy variables to represent the carriers' status in each market. For a particular market, two dummy variables are built to indicate the presence of carrier ${ }_{\mathrm{G}} i$ :

(i) $y_{i}^{c}$ equals to 1 if the carrier operates nonstop flights in the market and equals to 0 otherwise

(ii) $y_{i}^{c}$ equals to 1 if the carrier operates connecting flights in the market and equals to 0 otherwise

The analysis is focused on the strategic interactions among the top five carriers, namely, Southwest, American, Delta, United, and JetBlue (they are the top five carriers in 2017Q1, based on passenger count). The author then lumps the remaining LCCs into one composite carrier (including Spirit, Frontier, Allegiant, and Virgin), denoted as Small LCC, and creates a dummy variable $y_{\text {SLCC }}^{n}$ which equals to 1 if at least one of the small LCCs operates nonstop flights in the market, as well as a dummy variable $y_{\text {SLCC }}^{c}$ which equals to 1 if at least one of the small LCCs operates connecting flights in the market.

After the construction of dummy variables, the data are finally aggregated by market segments. For each market, there is a vector whose elements are all zeros and/or ones. This vector represents the structure of the market, that is, what carriers serve the market and what do not.

3.3. Market Selection. To select the markets, the author merges the aggregated dataset with demographic statistics obtained from the U.S. Census Bureau. The author first generates a ranking of airports by the metropolitan statistical area (MSA) population and keeps markets between the top 100 MSAs. After that, the author removes airports which have too few flights to the remaining airports. The final sample contains 3024 markets.

\section{Game Model and Estimation}

4.1. Payoff Functions. Closely following $[11,16]$, the author assumes that the basic payoff function for carrier $i$ in market $m$ is

$$
\pi_{i m}=\mathbf{S}_{\mathbf{m}}^{\prime} \boldsymbol{\alpha}+\mathbf{Z}_{\mathrm{im}}^{\prime} \boldsymbol{\beta}+\sum_{j \neq i} \delta_{j}^{i} y_{j m}+u_{i m},
$$

where $\mathbf{S}_{\mathbf{m}}$ is a vector of market characteristics which are common among all carriers in market $m ; \mathbf{Z}_{\mathbf{i m}}$ is a vector of carrier $i$ 's characteristics which enter only into carrier $i$ 's profit in market $m ; \mathbf{y}_{-\mathbf{i m}}$ is a vector that represents other carriers' presence in market $m$; and finally, $u_{i m}$ is the part of profits that are unobserved by economists.

The coefficients of interest are $\left\{\delta_{j}^{i}\right\}$, which summarize the impact of carrier $j$ 's presence on the payoff of carrier $i$. This effect is denoted as the "competitive effect." For example, the impact of Southwest's presence on Delta's payoff is captured by $\delta_{\mathrm{SW}}^{\mathrm{DL}}$, and the impact of Southwest's presence on JetBlue's profit is captured by $\delta_{\mathrm{SW}}^{\mathrm{JB}}$. 
Note that equation (1) does not take the type of service into consideration. When accounting for service types, the payoff function for carrier $i$ 's type $\varphi$ service in market $m$ is

$$
\pi_{i m}^{\varphi}=\mathbf{S}_{\mathbf{m}}^{\prime} \boldsymbol{\alpha}+\mathbf{Z}_{\mathrm{im}}^{\prime} \boldsymbol{\beta}+\delta_{i,-\varphi}^{i, \varphi} y_{i m}^{-\varphi}+\sum_{j \neq i} \delta_{j, \varphi}^{i, \varphi} y_{j m}^{\varphi}+\sum_{j \neq i} \delta_{j,-\varphi}^{i, \varphi} y_{j m}^{-\varphi}+u_{i m}^{\varphi},
$$

where $\varphi$ represents either nonstop service or connecting service. If $\varphi$ represents nonstop service, then $-\varphi$ represents the other type of service, namely, the connecting service, and vice versa. In this case, the competitive effects $\left\{\delta_{j, \varphi}^{i, \varphi}\right\}$ summarize the impact of carrier $j$ 's type $\varphi$ service on the payoff of carrier $i$ 's type $\varphi$ service, while $\left\{\delta_{j,-\varphi}^{i, \varphi}\right\}$ summarize the impact of carrier $j$ 's type $-\varphi$ service on the payoff of carrier $i$ 's type $\varphi$ service.

4.2. Variable Definitions. The variables used in this study are defined below.

The market characteristics contained in $\mathbf{S}_{\mathbf{m}}$ include the following:

(i) Market size: the geometric mean of population at the market endpoint MSAs

(ii) Per capita income: the average per capita income at the market endpoint MSAs

(iii) Income growth rate: the average rate of per capita income growth at the market endpoint MSAs

(iv) Market distance: the nonstop distance between the market endpoint airports

(v) Closest airport: the distance from each endpoint airport to the closest airport

(vi) Slot: a dummy variable that is equal to 1 if either one of the market endpoints is a slot-controlled airport, and is equal to 0 otherwise (there are four slotcontrolled airports in the US, including New York LaGuardia, New York Kennedy, Ronald Reagan Washington, and Chicago O'Hare)

The firm characteristics contained in $\mathbf{Z}_{\mathbf{i m}}$ include the following:

(i) Excess distance: the ratio of a carrier's minimum connecting distance in excess of nonstop distance over nonstop distance between the two endpoint airports. This variable can be interpreted as a proxy for the opportunity cost that a carrier has to face to operate nonstop flights in that market because it measures the cost of the best alternative to a nonstop flight, which is the shortest connecting flight.

(ii) Airport presence: a carrier's airport presence in a market is the average of its presence at the endpoint airports. For each carrier, its presence at a particular airport is computed as the number of markets served by it from/to the airport over the total number of markets from/to the airport. For example, let us consider Delta and the market between New York Kennedy and Chicago O'Hare. If Delta serves 40 out of New York Kennedy's 100 markets, then Delta's airport presence at New York Kennedy is 40/ $100=40 \%$. It is possible to perform the same calculation for Chicago O'Hare, let us say the airport presence at Chicago O'Hare is $25 \%$. Then, for the market between New York Kennedy and Chicago O'Hare, Delta's airport presence is $(40 \%+25 \%) /$ $2=32.5 \%$.

The dummy variables contained in $\left\{y_{i m}^{\varphi}\right\}$, as described in Section 3.2, are defined as follows:

(i) $y_{i m}^{n}$ equals to 1 if the carrier operates nonstop flights in the market and equals to 0 otherwise

(ii) $y_{i m}^{c}$ equals to 1 if the carrier operates connecting flights in the market and equals to 0 otherwise

The descriptive statistics for market characteristics variables and firm characteristics variables are summarized in Table 1.

4.3. The Game. The author constructs a static game of simultaneous entry. In doing so, it is assumed that, for each market, every carrier needs to simultaneously decide whether or not to enter it. A carrier will enter a particular market as long as it can realize nonnegative profit from entry. Its postentry profit in a market is specified as a function of market-level variables, of its own characteristics, and of the competitive effects brought by its competitors, as described in Section 4.1. Nash equilibrium is reached when in each market, every entered firm expects a nonnegative postentry profit and every unentered firm expects a negative postentry profit (for computational simplicity, only purestrategy Nash equilibria are accounted for).

4.4. Error Term. The error term in equation (2), $u_{i m}^{\varphi}$, consists of four parts: (i) a carrier-specific error term, $u_{i}$; (ii) a market-specific error term, $u_{m}$; (iii) two airport-specific error terms, $u_{o}$ and $u_{d}$; and (iv) a service-type-specific error term, $u_{\varphi} . u_{i m}^{\varphi}$ is computed by summing up those four parts.

The author assumes that $u_{i}, u_{m}, u_{o}, u_{d}$, and $u_{\varphi}$ are all independent and follow the standard normal distribution.

4.5. Algorithmic Implementation. The parameters in the payoff functions are estimated by searching for the set of parameter values which minimize the distance between the entry outcomes, predicted by the model Nash equilibrium, and the observed entry outcomes, shown in the data. The detailed procedure is as follows.

4.5.1. Objective Function. Assume that $\mathbf{X}=(\mathbf{S}, \mathbf{Z})$ represents the exogenous variables, and $\theta=(\alpha, \beta, \delta)$ represents the parameters to be estimated.

If there are $K$ potential carriers, then a particular equilibrium outcome $\mathbf{y}=\left(y_{1}^{n}, y_{1}^{c}, y_{2}^{n}, y_{2}^{c}, \ldots, y_{K}^{n}, y_{K}^{c}\right)$ is a sequence of zeroes and ones. Recall that each element in $\mathbf{y}$ is endogenous and determined by the expected profit, i.e., $y_{i}^{\varphi}=1\left[\pi_{i}^{\varphi}\left(\mathbf{X}, \theta, \mathbf{y}_{-\mathbf{i}}^{\varphi}, \mathbf{y}_{-\mathbf{i}}^{-\varphi}, y_{i}^{-\varphi}, u_{i}^{\varphi}\right) \geq 0\right], \forall i \in\{1, \ldots, K\}$, $\forall \varphi \in\{n, c\}$. 
Assume $\operatorname{Pr}(\mathbf{y} \mid \mathbf{X})$ is the probability function for outcome $\mathbf{y}$ given $\mathbf{X}$. The goal is to identify $\theta$ without explicitly specifying this function. Noticing that the probability function is bounded between 0 and 1 , one can further get

$$
\int_{R_{1}(\mathbf{X}, \boldsymbol{\theta})} d F(\mathbf{u}) \leq \operatorname{Pr}(\mathbf{y} \mid \mathbf{X}) \leq \underbrace{\int_{R_{1}(\mathbf{X}, \boldsymbol{\theta})} d F(\mathbf{u})}_{\text {unique outcome region }}+\underbrace{\int_{R_{2}(\mathbf{X}, \boldsymbol{\theta})} d F(\mathbf{u})}_{\text {multiple outcome region }},
$$

where $R_{1}$ is the region in which $\mathbf{y}$ is the unique observable outcome of the entry game and $R_{2}$ is the region in which the game admits multiple potentially observable outcomes, one of which is $\mathbf{y}$.

These bounds can then be written in a vector format as

$$
\mathbf{H}_{1}(\mathbf{X}, \boldsymbol{\theta}) \leq \operatorname{Pr}(\mathbf{y} \mid \mathbf{X}) \leq \mathbf{H}_{2}(\mathbf{X}, \boldsymbol{\theta}) .
$$

The model is estimated by ensuring that the observed probabilities of each equilibrium outcome for every $\mathbf{X}$ lie between the upper and lower bounds predicted by the model. The way to do this is by searching for the $\theta$ that minimizes

$$
\begin{aligned}
Q(\boldsymbol{\theta})= & \int\left[\left\|\operatorname{Pr}(\mathbf{y} \mid \mathbf{X})-\mathbf{H}_{1}(\mathbf{X}, \boldsymbol{\theta})\right\|_{-}\right. \\
& \left.+\left\|\operatorname{Pr}(\mathbf{y} \mid \mathbf{X})-\mathbf{H}_{2}(\mathbf{X}, \boldsymbol{\theta})\right\|_{+}\right] \mathrm{d} F_{x}(\mathbf{X}),
\end{aligned}
$$

which simply penalizes lower (upper) bounds predicted by the model that exceed (are less than) the observed probability of each equilibrium outcome.

In practice, $N$ points are drawn out of the distribution of $\mathrm{d} F_{x}(\mathbf{X})$, and the sample objective function is computed as

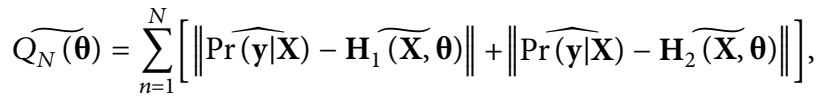

where $\operatorname{Pr} \widehat{(\mathbf{y} \mid \mathbf{X})}=\sum_{n=1}^{N} 1\left[\mathbf{y}_{\mathbf{n}}=\mathbf{y}\right] 1\left[\mathbf{X}_{\mathbf{n}}=\mathbf{X}\right] / \sum_{n=1}^{N} 1\left[\mathbf{X}_{\mathbf{n}}=\mathbf{X}\right]$ and $\left.\mathbf{H}_{1} \widetilde{(\mathbf{X}}, \theta\right)$ and $\left.\mathbf{H}_{2} \overline{(\mathbf{X}}, \theta\right)$ are simulated estimates of the lower and upper bounds predicted by the model.

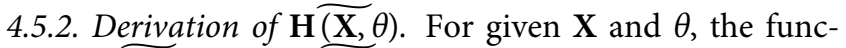
tions $\left.\mathbf{H}_{1} \overline{(\mathbf{X}}, \theta\right)$ and $\mathbf{H}_{2}(\mathbf{X}, \theta)$ are simulated as follows:

S1: set $\mathbf{H}_{1}(\mathbf{X}, \theta)=\mathbf{H}_{2}(\mathbf{X}, \theta)=0$.

S2: for each market, each firm, each origin/destination, and each service type, generate $R$ draws, respectively, from the normal distribution with an identity variancecovariance matrix. For each simulation $r=(1, \ldots, R)$, follow Steps 1-3:

Step 1. Transform the error term draws into a draw, and store it in $\mathbf{u}^{r}$.

Step 2. Calculate the vector of firms' profits for each set of entry decisions, $\mathbf{y}^{g}$ (for $g=1, \ldots, 2^{2 K}$ ):
TABle 1: Descriptive statistics.

\begin{tabular}{lccc}
\hline Variable & & Mean & St. dev. \\
\hline & American & 0.280 & 0.127 \\
& Delta & 0.339 & 0.160 \\
Airport presence & United & 0.212 & 0.101 \\
& Southwest & 0.352 & 0.200 \\
& JetBlue & 0.162 & 0.088 \\
& Small LCC & 0.209 & 0.177 \\
\hline & American & 0.506 & 1.232 \\
& Delta & 0.495 & 1.767 \\
& United & 0.672 & 1.633 \\
Excess distance & Southwest & 0.304 & 1.147 \\
& JetBlue & 0.423 & 0.970 \\
& Small LCC & 0.166 & 0.526 \\
\hline Market size (population) & & $3,026,113$ & $2,258,958$ \\
Per capita income (\$) & & $45,602.56$ & $4,210.65$ \\
Income growth rate (\%) & & 3.107 & 0.973 \\
Market distance (miles) & & 1031.617 & 578.920 \\
Closest airport (miles) & & 38.031 & 20.679 \\
Slot (0/1) & & 0.091 & 0.287 \\
Number of markets & & 3024 & \\
\hline
\end{tabular}

$$
\pi\left(\mathbf{y}^{g}, \mathbf{X}, \mathbf{u}^{r}, \boldsymbol{\theta}\right) \text {. }
$$

Step 3. Identify equilibria. For every $X(n=1, \ldots, N)$ and $\mathbf{u}^{r}(r=1, \ldots, R)$, the outcome $\mathbf{y}^{g}$ is an equilibrium if the vector $\pi\left(\mathbf{y}^{g}, X, \mathbf{u}^{r}, \theta\right) \geq 0$. For a unique equilibrium (that is, there is no $g^{\prime} \neq g$ such that $\left.\pi\left(\mathbf{y}^{g}, X, \mathbf{u}^{r}, \theta\right) \geq 0\right)$, set $\mathbf{H}_{1}^{g}=\mathbf{H}_{1}^{g}+(1 / R), \quad \mathbf{H}_{2}^{g}=\mathbf{H}_{2}^{g}+(1 / R)$. For a nonunique equilibrium, set $\mathbf{H}_{2}^{g}=\mathbf{H}_{2}^{g}+(1 / R)$.

This will yield the simulated versions of $\left.\mathbf{H}_{1} \widetilde{(\mathbf{X}}, \theta\right)$ and $\left.\mathbf{H}_{2} \widetilde{(\mathbf{X}}, \theta\right)$, which are then used to calculate the objective function, $\widetilde{Q_{N}(\theta)}$.

4.5.3. Construction of Confidence Region. Due to the existence of multiple equilibria, the model parameters might not be point identified. So instead of reporting point estimates, the author computes and reports a cube which is similar to the confidence region closely following $[11,24]$.

A key statistic for building this confidence region is

$$
C_{N}(c)=\left\{\theta \in \Theta: N\left(Q_{N}(\theta)-\min _{t} Q_{N}(t)\right) \leq c\right\},
$$

where $c$ is chosen as the level- $\alpha$ quantile of $C_{N}$. Keeping this in mind, the steps to calculate confidence region are as follows:

R1: minimize the objective function for the overall sample, and set $c_{0}$ as $25 \%$ above the overall minimum objective function value. This will be the starting cut off. R2: construct all subsets of size $b \ll N$. Suppose the total number of subsets is $B_{N}$. For every $i$ th subset, $i \leq B_{N}$, minimize the subset objective function and then 
compute $C_{i, c_{0}}=\sup _{t \in C_{N}\left(c_{0}\right)} b\left(Q_{b}(t)-\min _{t} Q_{b}(t)\right)$, where $C_{N}\left(c_{0}\right)=\left\{\theta \in \Theta: N\left(Q_{N}(\theta)-\min _{t} Q_{N}(t)\right) \leq c_{0}\right\}$.

R3: obtain $\sup _{i \leq B_{N}} C_{i, c_{0}}$, and set its level- $\alpha$ quantile as $c_{1}$. This will be the new cut off.

$\mathrm{R} 4$ : repeat steps $\mathrm{R} 2$ and $\mathrm{R} 3$, replacing $c_{0}$ with $c_{1}$. This will give us $c_{2}$.

R5: obtain $C_{N}\left(c_{2}\right)=\left\{\theta \in \Theta: N\left(Q_{N}(\theta)-\min _{t} Q_{N}(t)\right)\right.$ $\left.\leq c_{2}\right\}$. This will be the confidence region to be reported (for proofs that this is an asymptotically equivalent confidence region, please see $[11,24]$.

4.5.4. Computing Time. The computation is carried out in the cluster with the configuration of each node with 2.00 GHz Intel Core i7-9700T CPU. Setting $R=50$, Table 2 summarizes the average computing time as the sample size $(N)$ and subsample size $(b)$ vary. From the table, it is clear that as $N$ increases, the computing time increases exponentially, mainly because the process requires an optimization step at each subsample.

\section{Results and Discussion}

5.1. Competitive Effects. Table 3 reports the estimates of competitive effects when setting $R=50$ and $b=600 \approx(N / 5)$. The negative sign means that the presence of Southwest has a negative impact on the carrier's profit, and the numbers in the square brackets indicate to what extent the carrier's profit declines with the presence of Southwest. Recall that the profit here is an index recovered from the game and is not true profit in dollar value. However, the changes of the index should be able to reveal the changes of the true profit.

In column (I), it is assumed that Southwest's competitive effects on non-Southwest carriers are the same, i.e., American, Delta, United, and JetBlue, and the remaining small LCCs bear the same competitive effects from Southwest. The results show that Southwest's nonstop presence affects non-Southwest carriers' nonstop profits by [-25.48, -21.19], which is larger than its impact on non-Southwest carriers' connecting profits $([-16.73,-12.79])$. The reason may be that Southwest's nonstop service competes head to head with non-Southwest carriers' nonstop services, but only partially with their connecting services. Similarly, Southwest's connecting presence affects non-Southwest carriers' connecting profits by $[-7.67,-5.01]$, which is more significant than its impact on non-Southwest carriers' nonstop profits $([-2.63,-0.02])$, possibly because the competition between connecting services is head to head whereas the competition between connecting and nonstop services is not. Furthermore, it is clear that the competitive effects of Southwest's nonstop service are much larger than those of its connecting service. Viewing the nonstop service as a higher-quality product compared to the connecting service, this result implies that the quality of a product is a crucial determinant of its competitive effects, where a
TABLE 2: The average computing time.

\begin{tabular}{llc}
\hline$N$ & $\mathrm{~b}$ & Average computing time (hours) \\
\hline \multirow{2}{*}{300} & $N / 6$ & 3.39 \\
& $N / 5$ & 5.15 \\
\hline \multirow{2}{*}{600} & $N / 6$ & 9.59 \\
& $N / 5$ & 14.34 \\
\hline \multirow{2}{*}{900} & $N / 6$ & 24.77 \\
& $N / 5$ & 38.68 \\
\hline \multirow{2}{*}{1200} & $N / 6$ & 58.89 \\
& $N / 5$ & 81.27 \\
\multirow{2}{*}{1500} & $N / 6$ & 142.71 \\
& $N / 5$ & 196.44 \\
\hline
\end{tabular}

higher-quality product would put a lot more downward pressure on opponents' profits.

In column (II), the author allows Southwest's competitive effects on various carriers to be different. In this scenario, the conclusions obtained from column (I) are still valid. Besides, the magnitude indicates that FSCs are less affected by Southwest compared with LCCs. Among LCCs, small LCC is slightly more impacted than big LCC (JetBlue), except for the connect-on-connect case where JetBlue is a little bit more affected.

Regarding the exogenous variables, the results show that excess distance and slot control have negative effects on carrier's profit, while airport presence, per capita income, income growth rate, and market size have positive effects on the carrier's profit.

Comparing the two columns, there are some differences in the signs of market distance and closest airport. For example, in column (I) the coefficient of market distance is $[-1.77,0.89]$, whereas in column (II), the coefficient is [1.02, 2.05]. Combining with the finding in [25] that flight distance is positively related to profitability, this result suggests that the assumption of a homogeneous competitive effect of Southwest, on various opponents, may lead to some bias in estimation.

For robustness check, the simulation is redone by setting $b=500 \approx(N / 6)$. The estimates for competitive effects are summarized in Table 4. Comparing Tables 3 and 4, the patterns of competitive effects are essentially the same, which enhances the author's confidence on the estimation results.

5.2. Counterfactual Experiment. To further assess Southwest's impacts, the author conducts a counterfactual experiment to investigate how carriers' entry probabilities may change when Southwest is counterfactually removed. If the change is positive, i.e., the entry probability of a carrier increases when Southwest is eliminated, then the entry of this carrier should be discouraged by Southwest. The larger the change of probability, the more the Southwest affects the carrier's entry decision.

Essentially, the author performs the counterfactual by setting the competitive effects of Southwest equivalent to zero (hence "removing" Southwest from each market), computing the new equilibria, and then, comparing the equilibria with Southwest being removed to the initial 
TABLE 3: Model results of competitive effects.

\begin{tabular}{|c|c|c|}
\hline & (I) & (II) \\
\hline \multicolumn{3}{|l|}{ Competitive effect } \\
\hline $\begin{array}{l}\text { SW nonstop on non-SW } \\
\text { nonstop }\end{array}$ & $\begin{array}{l}{[-25.48} \\
-21.19]\end{array}$ & \\
\hline $\begin{array}{l}\text { SW nonstop on non-SW } \\
\text { connect }\end{array}$ & $\begin{array}{l}{[-16.73} \\
-12.79]\end{array}$ & \\
\hline $\begin{array}{l}\text { SW connect on non-SW } \\
\text { nonstop }\end{array}$ & {$[-2.63,-0.02]$} & \\
\hline $\begin{array}{l}\text { SW connect on non-SW } \\
\text { connect }\end{array}$ & {$[-7.67,-5.01]$} & \\
\hline SW nonstop on FSC nonstop & & $\begin{array}{l}{[-18.13,} \\
-13.95]\end{array}$ \\
\hline \multicolumn{3}{|l|}{ FSCs: $A A, D L$, and $U A$} \\
\hline SW nonstop on JetBlue nonstop & & $\begin{array}{l}{[-23.25} \\
-19.20]\end{array}$ \\
\hline $\begin{array}{l}\text { SW nonstop on small LCC } \\
\text { nonstop }\end{array}$ & & $\begin{array}{l}{[-26.98} \\
-23.18]\end{array}$ \\
\hline SW nonstop on FSC connect & & $\begin{array}{l}{[-13.26} \\
-10.02]\end{array}$ \\
\hline SW nonstop on JetBlue connect & & $\begin{array}{l}{[-17.05} \\
-13.13]\end{array}$ \\
\hline $\begin{array}{l}\text { SW nonstop on small LCC } \\
\text { connect }\end{array}$ & & $\begin{array}{l}{[-18.98} \\
-14.74]\end{array}$ \\
\hline SW connect on FSC nonstop & & {$[-1.03,0.91]$} \\
\hline SW connect on JetBlue nonstop & & {$[-2.14,-0.47]$} \\
\hline $\begin{array}{l}\text { SW connect on small LCC } \\
\text { nonstop }\end{array}$ & & {$[-2.57,-1.30]$} \\
\hline SW connect on FSC connect & & {$[-4.89,-3.91]$} \\
\hline SW connect on JetBlue connect & & {$[-7.47,-5.03]$} \\
\hline $\begin{array}{l}\text { SW connect on small LCC } \\
\text { connect }\end{array}$ & & {$[-6.82,-4.95]$} \\
\hline Excess distance & {$[-0.25,-0.03]$} & {$[-0.81,-0.41]$} \\
\hline Airport presence & {$[0.18,0.22]$} & {$[0.35,0.47]$} \\
\hline Per capita income & {$[2.47,2.91]$} & {$[5.44,5.89]$} \\
\hline Income growth rate & {$[0.52,0.72]$} & {$[0.63,1.17]$} \\
\hline Slot control & {$[-2.69,-2.44]$} & {$[-3.24,-2.13]$} \\
\hline Market size & {$[5.11,5.89]$} & {$[6.98,8.86]$} \\
\hline Market distance & {$[-1.77,0.89]$} & {$[1.02,2.05]$} \\
\hline Closest airport & {$[-1.04,0.44]$} & {$[-2.68,-1.99]$} \\
\hline Constant & {$[-10.60,-8.14]$} & {$[-8.60,-6.15]$} \\
\hline
\end{tabular}

Note. The intervals reported in this table can be interpreted as the $95 \%$ confidence region. "SW" represents Southwest. "Non-SW" represents nonSouthwest carriers. "FSC" refers to major full-service carriers, including American, Delta, and United. "Small LCC" refers to small low-cost carriers, including Spirit, Frontier, Allegiant, and Virgin. The simulation is performed by setting $R=50$ and $b=600$.

equilibria where Southwest operates (the parameter values in column (II) in Table 3 are used to compute equilibria. To be specific, the middle points of the confidence intervals are used. For example, the airport presence coefficient estimate is $[0.35,0.47]$; then, the author uses 0.41 (the middle point of 0.35 and 0.47 ) to compute equilibria). Due to the existence of multiple equilibria, the author only observes the lower and upper bounds on the entry probability of each carrier. So, following [11], the author records the changes of upper
TABLE 4: Robustness check.

\begin{tabular}{lcc}
\hline Competitive effect & (I) & (II) \\
\hline SW nonstop on non-SW & {$[-24.20$,} & \\
nonstop & $-20.52]$ & \\
SW nonstop on non-SW & {$[-14.57$,} & \\
connect & $-11.56]$ & \\
SW connect on non-SW & {$[-2.95,-0.49]$} & \\
nonstop & & \\
SW connect on non-SW & {$[-5.89,-4.78]$} & \\
connect & & {$[-18.56$,} \\
SW nonstop on FSC nonstop & $-14.70]$ \\
\hline FSCs: $A A$, DL, and UA & & \\
SW nonstop on JetBlue nonstop & & $-18.76]$ \\
SW nonstop on small LCC & {$[-28.39$,} \\
nonstop & $-24.02]$ \\
SW nonstop on FSC connect & {$[-12.02$,} \\
& $-10.55]$ \\
SW nonstop on JetBlue connect & {$[-16.76$,} \\
SW nonstop on small LCC & $-12.51]$ \\
connect & {$[-18.12$,} \\
SW connect on FSC nonstop & $-13.66]$ \\
SW connect on JetBlue nonstop & {$[-0.84,0.94]$} \\
SW connect on small LCC & {$[-2.93,-1.10]$} \\
nonstop & {$[-3.35,-1.72]$} \\
SW connect on FSC connect & {$[-4.05,-3.23]$} \\
SW connect on JetBlue connect & \\
SW connect on small LCC & {$[-6.60,-6.15]$} \\
connect & \\
Note. For robustness check, the simulation is redone by setting $b=500$. \\
\end{tabular}

bounds for a given carrier and reports the biggest positive change along with the median change. The results are summarized in Table 5.

According to the results, for each carrier, the change in the mean upper bounds is on the positive side, indicating that Southwest discourages the entries of them. Once Southwest is eliminated, each carrier is more likely to enter a market.

To be specific, in the case of nonstop service, the likelihood of small LCC entering a market boosts by up to $41.1 \%$ when Southwest is removed, with a median of $18.2 \%$. This is the largest change across all carriers. On the other hand, FSCs are least affected by Southwest, where the entry probability changes by up to $30.4 \%$, with a median of $12.3 \%$. In the case of connecting service, FSCs again exhibit the smallest change (up to $19.5 \%$, median of $7.1 \%$ ). Big LCC (JetBlue) and small LCC show similar changes, where the medians are $11.6 \%$ and $11.0 \%$, respectively. In addition, comparing the two service types, it is apparent that nonstop services display much greater changes, indicating that carriers' nonstop entries are more impacted by Southwest than their connecting entries. 
TABLE 5: Changes in entry probabilities when Southwest is counterfactually removed.

\begin{tabular}{lcc}
\hline Airline & $\begin{array}{c}\text { Biggest positive change } \\
(\%)\end{array}$ & $\begin{array}{c}\text { Median change } \\
(\%)\end{array}$ \\
\hline FSC nonstop & 30.4 & 12.3 \\
\hline FSCs: $A A, D L$, and UA & & \\
$\begin{array}{l}\text { FSC connect } \\
\text { JetBlue nonstop }\end{array}$ & 19.5 & 7.1 \\
JetBlue connect & 36.0 & 16.4 \\
$\begin{array}{l}\text { Small LCC } \\
\text { nonstop }\end{array}$ & 24.4 & 11.6 \\
$\begin{array}{l}\text { Small LCC } \\
\text { connect }\end{array}$ & 41.1 & 18.2 \\
\hline
\end{tabular}

Note. The author reports the biggest positive change and the median change in the upper bounds of entry probabilities. The parameter values in column (II) in Table 3 are used to calculate.

\section{Conclusions and Discussion}

This paper studies the effects of Southwest Airlines on the profits and entry probabilities of other carriers in the U.S. air travel market. The author constructs a static game of simultaneous entry similar to [10] and then estimates the parameters using the bounds method proposed in [11]. The results show that Southwest has a notable, negative impact on the profits of other carriers. In particular, the profits of LCCs, including JetBlue and small LCC, are more impacted than those of FSCs. Comparing the two service types, the findings show that Southwest's nonstop service imposes much more downward pressure on other carriers' profits than its connecting service. Finally, through counterfactual experiment, the author confirms that Southwest has a remarkable impact on the entry probability of each carrier.

This paper extends literature on LCCs by investigating the effects of Southwest from a new perspective. Though massive research studies have been conducted to assess the effects of LCCs on airfares and passenger traffic, their impacts on profits or entry probabilities are rarely examined. The present paper addresses this matter and fills the gap. Meanwhile, this paper also extends literature on the empirical analysis of entry games, by being the first work to incorporate both nonstop and connecting services into the model.

The conclusions in this paper have policy implications for the antitrust regulators. In airline antitrust practices, a disputable question is whether nonstop and connecting products are effectively competing with each other. The findings of this work indicate that nonstop products put substantial pressure on connecting products, but not the converse, hence shedding light on this question.

More generally, the results in this article provide some clue into what one might expect to find in other vertically differentiated product markets. When products differ in quality, a higher quality product may generate much larger competitive effects than a lower quality one; or, to put it in another way, the quality of a product might be an essential factor in the analysis of competition.

To enable readers to interpret the results accurately, it is necessary to recognize the limitations of this study. First, for simplicity, the author only examines the one-period static equilibrium. Future research may want to extend the analysis to multiple periods and see whether the results are still valid in the case of repeated games. Second, the author assumes that each carrier takes its entire network as well as the networks of its competitors as given. The network effect is also not considered in the model. Future research may account for the network effect to obtain more interesting results. Third, the computing time of the game increases exponentially as the sample size increases, indicating that the proposed framework may not be feasible on large samples. It would be ideal if one could improve the estimation process so that it could work on large datasets.

\section{Data Availability}

The data used to support the findings of this study are available from the author upon reasonable request.

\section{Conflicts of Interest}

The author declares no conflicts of interest.

\section{Acknowledgments}

The author would like to appreciate Professor Jason R. Blevins from the Ohio State University for his valuable comments and assistance.

\section{References}

[1] J. Ren, "Fare impacts of Southwest airlines: a comparison of nonstop and connecting flights," Journal of Air Transport Management, vol. 84, Article ID 101771, 2020.

[2] M. B. Abda, P. P. Belobaba, and W. S. Swelbar, "Impacts of LCC growth on domestic traffic and fares at largest US airports," Journal of Air Transport Management, vol. 18, no. 1, pp. 21-25, 2012.

[3] R. Asahi and H. Murakami, "Effects of Southwest Airlines' entry and airport dominance," Journal of Air Transport Management, vol. 64, pp. 86-90, 2017.

[4] M. Dresner, J. S. C. Lin, and R. Windle, "The impact of lowcost carriers on airport and route competition," Journal of Transport Economics and Policy, vol. 30, no. 3, pp. 309-328, 1996.

[5] A. Goolsbee and C. Syverson, "How do incumbents respond to the threat of entry? Evidence from the major airlines*," Quarterly Journal of Economics, vol. 123, no. 4, pp. 1611-1633, 2008.

[6] J. Kwoka and B. Batkeyev, "Strategic responses to competitive threats: airlines in action," Review of Industrial Organization, vol. 54, no. 1, pp. 83-109, 2019.

[7] S. A. Morrison, "Actual, adjacent, and potential competition estimating the full effect of Southwest Airlines," Journal of Transport Economics and Policy, vol. 35, no. 2, pp. 239-256, 2001.

[8] A. V. M. Oliveira and C. Huse, "Localized competitive advantage and price reactions to entry: full-service vs. low-cost airlines in recently liberalized emerging markets," Transportation Research Part E: Logistics and Transportation Review, vol. 45, no. 2, pp. 307-320, 2009. 
[9] R. Windle and M. Dresner, "Competitive responses to low cost carrier entry," Transportation Research Part E: Logistics and Transportation Review, vol. 35, no. 1, pp. 59-75, 1999.

[10] Y. Zhang and M. Guizani, Game Theory for Wireless Communications and Networking, CRC Press, Boca Raton, FL, USA, 2011.

[11] F. Ciliberto and E. Tamer, "Market structure and multiple equilibria in airline markets," Econometrica, vol. 77, no. 6, pp. 1791-1828, 2009.

[12] K. Richards, "The effect of Southwest airlines on U.S. Airline markets," Research in Transportation Economics, vol. 4, pp. 33-47, 1996.

[13] R. D. Bennett and J. M. Craun, The Airline Deregulation Evolution Continues: The Southwest Effect, US Department of Transportation, Washington, DC, USA, 1993.

[14] R. Windle and M. Dresner, "The short and long run effects of entry on U.S. domestic air routes," Transportation Journal, vol. 35, no. 2, pp. 14-25, 1995.

[15] H. Murakami, "Time effect of low-cost carrier entry and social welfare in US large air markets," Transportation Research Part E: Logistics and Transportation Review, vol. 47, no. 3, pp. 306-314, 2011.

[16] S. T. Berry, "Estimation of a model of entry in the airline industry," Econometrica, vol. 60, no. 4, pp. 889-917, 1992.

[17] T. F. Bresnahan and P. C. Reiss, "Entry in monopoly markets," The Review of Economic Studies, vol. 57, no. 4, pp. 531-553, 1990.

[18] T. F. Bresnahan and P. C. Reiss, "Empirical models of discrete games," Journal of Econometrics, vol. 48, no. 1-2, pp. 57-81, 1991.

[19] P. C. Reiss and P. T. Spiller, "Competition and entry in small airline markets," The Journal of Law and Economics, vol. 32, no. 2, pp. 179-202, 1989.

[20] A. Dunn, "Do low-quality products affect high-quality entry? Multiproduct firms and nonstop entry in airline markets," International Journal of Industrial Organization, vol. 26, no. 5, pp. 1074-1089, 2008.

[21] P. G. Gayle and C. Y. Wu, "Are Air Travel Markets Segmented along the Lines of Nonstop versus Intermediate-Stop(s) Products?" Manuscript, Kansas State University, Manhattan, KS, USA, 2011.

[22] K. M. Tan, "Incumbent response to entry by low-cost carriers in the U.S. Airline industry," Southern Economic Journal, vol. 82, no. 3, pp. 874-892, 2016.

[23] S. Borenstein, "Hubs and high fares: dominance and market power in the U.S. airline industry," The RAND Journal of Economics, vol. 20, no. 3, pp. 344-365, 1989.

[24] V. Chernozhukov, H. Hong, and E. Tamer, "Estimation and confidence regions for parameter sets in econometric models," Econometrica, vol. 75, no. 5, pp. 1243-1284, 2007.

[25] A. V. M. Oliveira, "An empirical model of low-cost carrier entry," Transportation Research Part A: Policy and Practice, vol. 42, no. 4, pp. 673-695, 2008. 